\section{Repensar las ciencias y las fronteras disciplinares. Reflexiones a partir de proyectos de extensión sobre emergencia hídrica de la Universidad del Litoral}

\author{
Anabella Córdoba \\ Docente extensionista de la \\ Universidad Tecnológica Nacional \\ y de la Universidad Nacional del \\ Litoral, Argentina. \\ Coordinadora de operatorias de \\ emergencias hídricas. Ministerio de \\ Infraestructura y Transporte de la \\ Provincia de Santa Fe, Argentina. \\ anabellacordoba2003@gmail.com
}

\author{
María del Valle Morresi \\ Docente de la Facultad de Ingeniería \\ y Ciencias Hídricas, Universidad \\ Nacional del Litoral. \\ valle@fich.unl.edu.ar

\section{Patricia Chialvo} \\ Docente de la Facultad de Ciencias \\ Jurídicas y Sociales, Universidad \\ Nacional del Litoral. \\ patricia_chialvo@hotmail.com
}

\section{Resumen}

Este artículo aborda la articulación interdisciplinaria e interinstitucional de docentes y estudiantes universitarios respecto de la problemática de la emergencia hídrica a partir de proyectos de extensión de interés social que se vienen desarrollando en la Universidad Nacional del Litoral desde hace varios años.

Parte de comprender a la educación, en su más amplia acepción, como la transmisión de la cultura de una a otra generación, como el espacio en que el sujeto entra en contacto con la experiencia humana y la apropia, es decir aprende. Cada sujeto va haciendo suya su cultura a partir de procesos de aprendizajes significativos y situados, que le permiten el dominio progresivo de los objetos, de modos de pensar, sentir, actuar legitimados en cada contexto histórico.

En este marco nos propusimos articular las ciencias duras con las ciencias sociales y jurídicas mediante la recuperación y el reconocimiento de los diferentes tránsitos, historias y desarrollos que han tenido. En este sentido, nos dimos cuenta de que en las últimas décadas, y a partir de la temática que nos convoca, el riesgo es constitutivo de la sociedad en que vivimos en la actualidad, a la que intentamos comprender desde el paradigma de la complejidad en una permanente articulación dinámica y posible.

\section{Palabras clave}

- Interdisciplinariedad

- Interinstitucionalidad

- Educación

- Extensión
Integración de la docencia y la extensión /

Perspectivas

RECEPCIÓN: 24/06/16

ACEPTACIÓN FINAL: 10/10/16

\section{Resumo}

Este artigo discute a coordenação interdisciplinar e interinstitucional de professores e estudantes universitários para abordar o problema da emergência hídrica a partir de projetos de extensão de interesse social que se vêm desenvolvendo há vários anos.

Parte de compreender a educação, em sua acepção mais ampla, como a transmissão da cultura de uma geração para outra, como o espaço em que o sujeito entra em contato com a experiência humana e a sua própria, é dizer aprende. Cada pessoa vai empossar-se da sua própria cultura a partir de processos de aprendizagem significativos e situados, que the permitem o domínio progressivo dos objetos, de formas de pensar, sentir, agir legitimados em cada contexto histórico.

Nesta conjuntura, propusemo-nos articular as ciências duras com as ciências sociais e jurídicas através da recuperação e do reconhecimento dos diferentes trajetos, histórias e crescimentos que tiveram. Nesse sentido, percebemos que nas últimas décadas, e a partir do assunto que nos convoca, o risco é constitutivo da sociedade em que vivemos atualmente, que tentamos entendê-la desde o paradigma da complexidade em uma permanente articulação dinâmica e possível.

Palavras-chave

- Interdisciplinaridade

- Cooperação interinstitucional

- Educação

- Extensão

\section{Para citación de este artículo}

Córdoba, A. Morresi, M. V. y Chialvo, P. (2016). Repensar las ciencias y las fronteras disciplinares. Reflexiones a partir de proyectos de extensión sobre emergencia hídrica de la Universidad del Litoral. En Revista +E versión digital, (6), pp. 42-49. Santa Fe, Argentina: Ediciones UNL. 


\section{Introducción}

Situados en el presente siglo, nos encontramos inmersos en un escenario de profundos cambios en nuestra estructura sociopolítica, socioeconómica, cultural y relacional.

Desde estas condiciones de existencia consideramos que no se está frente a una simple condición que favorece u obstaculiza el aprendizaje y desarrollo del sujeto, sino que es una parte intrínseca del propio proceso, define su esencia. Por lo dicho, educación, aprendizaje y desarrollo humano son procesos que poseen una relativa singularidad pero que se integran al mismo tiempo conformando una unidad dialéctica.

Es por ello que la Universidad se convierte en el contexto por excelencia para estimular el desarrollo integral del estudiante en tanto se logren, como plantea Sacristán (citado por Córdoba, 2007a:32) "abordajes desde visiones totalizadoras capaces de alcanzar interpretaciones más profundas y anticipaciones más pertinentes". Y continúa: "Las limitaciones personales y profesionales de la especialización sólo pueden compensarse con las cualidades del trabajo en equipo de los practicantes de diversas disciplinas" (34). Esto es posible a través de prácticas de enseñanza y aprendizaje interdisciplinarias, que al decir de Carballeda: "se definen como la confrontación en el diálogo de distintas visiones de mundo traducidas en conocimiento" (2007b:1).

Según Apostel, Briggs y Guy Michaud:

"es la interacción existente entre dos o más disciplinas, que mantienen diversos canales de comunicación. Esta interacción puede ir de la simple comunicación de ideas hasta la integración mutua de conceptos directores, de la epistemología, de la terminología, de la metodología, de los procesos, de los datos y la organización de la investigación y de la enseñanza correspondiente". (1972:23-24)

En este marco desde dos Facultades -Ciencias Hídricas y Ciencias Jurídicas y Sociales - tres disciplinas (Ingeniería en Recursos Hídricos, Licenciatura en Trabajo Social y Abogacía) vienen trabajando desde 2010 en proyectos pertenecientes al Programa Ambiente y Sociedad que articulan docencia y extensión universitaria: "El recurso hídrico en hábitat vulnerable de la ciudad de Santa Fe" (2014-2016); "Fortalecimiento de roles para le gestión de la información hídrica" (2012-2014) y Red de capacitación para gestión de la información hídrica de la ciudad de Santa Fe" (2010-2012).

Este artículo abordará la base conceptual y metodológica para la construcción colectiva interdisciplinaria e intersectorial de conocimientos y saberes relacionados con un eje disciplinar o línea prioritaria seleccionada. Se presenta una propuesta concreta desde lo experiencial.

\section{La intervención en las instituciones de educación superior} Históricamente, la universidad tuvo una organización de la enseñanza basada en la división del conocimiento en disciplinas. Éstas constituyen la base de la estructura académica y como tal fueron limitando el ámbito de la reflexión teórica al fijar límites epistemológicos en el terreno de la formación disciplinaria y de la organización obstaculizando la comunicación entre ellas. Las fronteras disciplinarias, sus lenguajes, sus conceptos propios y sus prácticas, fueron aislando la disciplina respecto de las otras y de los problemas que las entrelazan, atomizándose en la especificidad.

En este sentido, acordamos con lo que plantea Morin:

"la disciplina es una categoría organizadora dentro del conocimiento científico; instituye en éste la división y la especialización del trabajo y responde a la diversidad de dominios que recubren las ciencias. Por más que esté inserta en un conjunto científico más vasto, una disciplina tiende, naturalmente, a la autonomía, por medio de la delimitación de sus fronteras, por el lenguaje que se da, por las técnicas que tiene que elaborar o utilizar y, eventualmente, por las teorías propias". (2004:115)

Es por ello que la universidad ha ido buscando constantemente nuevas formas de organizar la enseñanza, la investigación y la 


\section{6}

las fronteras disciplinarias, sus lenguajes, sus conceptos propios y sus prácticas, fueron aislando la disciplina respecto de las otras y de los problemas que las entrelazan

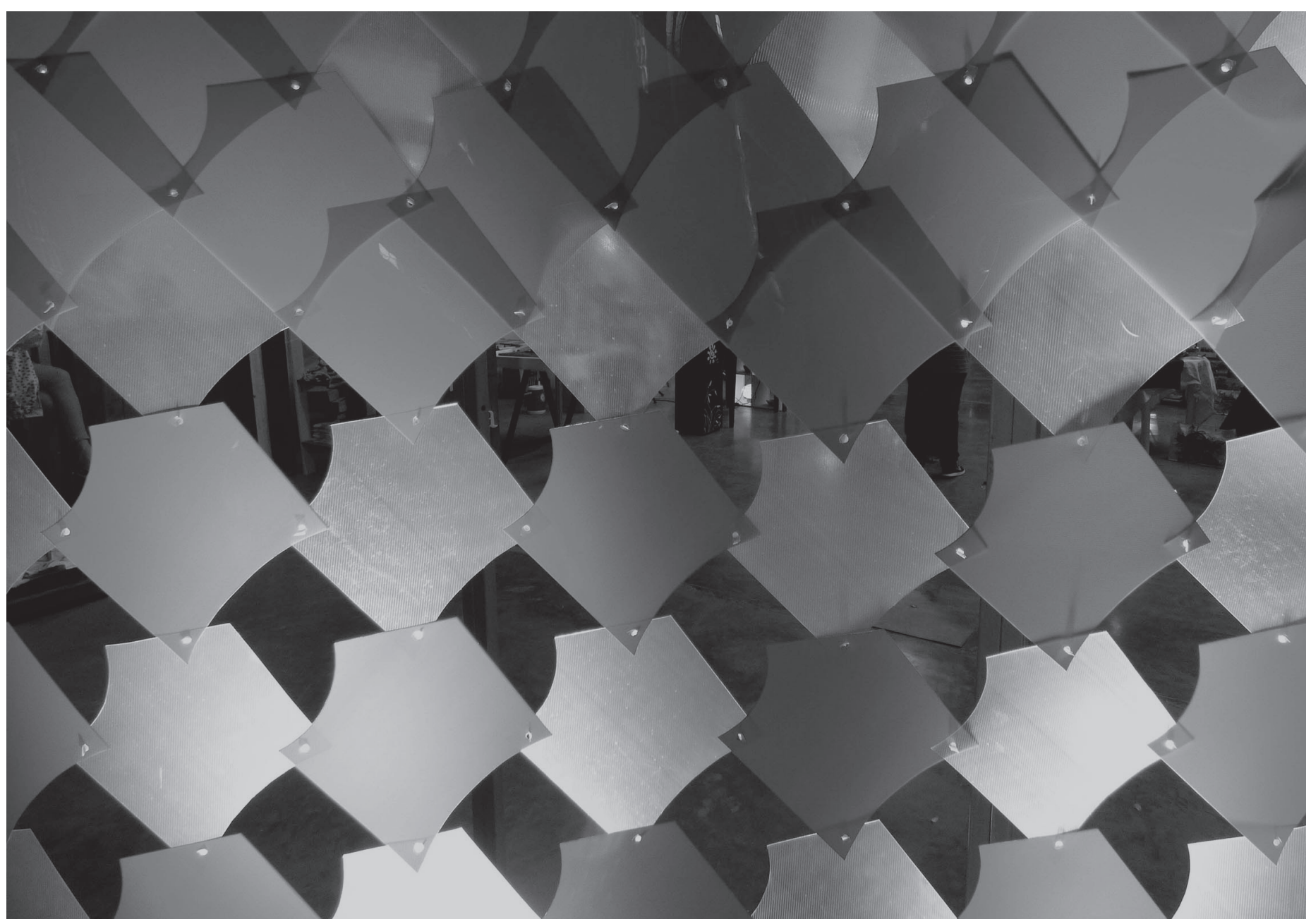

(C) Micaela Block 
extensión a través de diálogos entre disciplinas, para responder a los retos científicos ${ }^{1}$ y a las problemáticas sociales de modo de proporcionar una comprensión integral de la realidad.

En la Universidad Nacional del Litoral se ha producido tanto en el terreno teórico como en el práctico una cantidad considerable de propuestas para dar respuesta a este cuestionamiento. Los enfoques son diversos: unos parten de la noción de ciencia, otros de la forma en que se organiza la disciplina científica y otros del proceso de institucionalización de la enseñanza y del aprendizaje universitario. El debate en torno a esta cuestión y a la necesidad de la interdisciplinariedad se genera tanto a nivel cognoscitivo, pedagógico, sociocultural, en la producción y en su enseñanza en contraposición con la fragmentación y el dominio de la especialización que limitan la integración del saber y la construcción del conocimiento general. En este sentido, es importante mencionar que partimos de una mirada que no niega lo disciplinar y endiosa la interdisciplina, sino que decimos que ésta debe trascender la mirada reduccionista e ir integrando críticamente los avances que desde los campos disciplinares se han ido logrando. Al respecto Follari expresa que la interdisciplina:

"es complementaria al enfoque disciplinario; hace emerger de la confrontación de las disciplinas nuevos datos que las articulan entre sí, y nos ofrece una nueva visión de la naturaleza y de la realidad. No busca el dominio de muchas disciplinas, sino la apertura de todas las disciplinas a aquellos que las atraviesan y las trascienden". (2008:122)

La producción de prácticas de enseñanza y aprendizaje, con el principio ontológico de la interdisciplinariedad, parte de comprender este proceso como una praxis dialéctica dirigida por un interés emancipador y transformador de los sujetos. Por ello se propicia la interactuación permanentemente de las disciplinas, desde un programa amplio que les permita conocer, relacionar las diferentes lecturas del objeto de estudio, del sitio de trabajo, etcétera. Es de destacar que, el prefijo "inter" hace alusión a relaciones recíprocas, actividades de cooperación: interdependencia, intercambio e interpenetración. De este modo, podemos comprender que las referencias a las actividades inter o transdisciplinarias sugieren que son dinámicas interactivas que tienen como consecuencia una transformación recíproca de las disciplinas relacionadas con el campo/sujeto/objeto/contexto determinado. Del Percio incorpora otro concepto: la "indisciplina" como una aplicación de la transdisciplina, tomando el prefijo "in" en un triple sentido: como "entrar" desde una disciplina en otra, como "poner" otra disciplina en aquella desde la que se parte y como negación de los paradigmas de cada disciplina cuando ello fuere indispensable:

"palabra que connota una cierta disconformidad, una rebeldía frente al estado de cosas; es decir, hace referencia a esa cuestión del poder y alude a una suerte de resistencia frente a las visiones dominantes de la ciencia" (2009:3).

Desde esta perspectiva, la interdisciplina es considerada un camino para trabajar brindando soluciones integrales a las problemáticas planteadas, incorporando dinámicamente la mirada y visión del otro sujeto social individual, grupal o colectivo, destinatario de las políticas públicas, decisor, mediador, etc. Al considerar la visión del implicado, estamos dando respuestas a un contexto, a una problemática que tiene un anclaje territorial, que es dinámica, cambiante y requiere de respuestas interdisciplinares adecuadas a estos cambios.

Comprenderlo como proceso abierto nos permite darnos cuenta de que en este camino hay diversos niveles de interacción a alcanzar. De acuerdo con Peñuela Velázquez (citado por Arias Alpízar, 2009) existen:

a) La interdisciplinariedad lineal tipo 1: varias disciplinas abordan un mismo problema u objeto de estudio y cada una aporta desde su saber elementos para una mejor comprensión, pero ninguna sufre cambios o modificaciones determinables; el problema es el centro de la acción.

b) La interdisciplinariedad lineal tipo 2: una disciplina se apoya en algún elemento de otra (teoría, técnica-método, una información, un concepto), pero cada una conserva sus límites y dinámicas; la disciplina 2 puede, o no, verse afectada por la disciplina 1 y viceversa.

c) La interdisciplinariedad dialéctica: tanto la disciplina 1 como la disciplina 2 se afectan y cambian recíprocamente; hay interacción, intercambio y cooperación; lo que determina el intercambio varía de una tipología a otra.

d) El nivel dialéctico fractal simple: existe la emergencia de una nueva disciplina como emergencia de la interacción de otras disciplinas; la nueva disciplina no puede explicarse por la suma de las disciplinas que le dan origen (principios de sinergia y recursividad).

e) El nivel dialéctico fractal complejo: en él se dan niveles intermedios (fraccionarios), que son niveles complejos de interacción y donde se requieren altos niveles de conceptualización; la dimensión práctica solo puede ser aprehendida a partir de la interpretación en varios niveles de realidad y de esquemas cognitivos constructivos (no lineales, multicausales y azarosos). 
Como podemos apreciar, es sumamente complejo y estamos frente a un gran desafío ya que implica asumir cambios en los modos de mirar, percibir, abordar el trabajo académico.

Consideramos que esta propuesta pedagógica es un aporte sustantivo a la formación puesto que, al decir de Ferry, "formarse es objetivarse y subjetivarse en un movimiento dialéctico que va siempre más allá, más lejos" (1997:54).

\section{Compartiendo nuestra experiencia}

La ciudad de Santa Fe tiene la singularidad de haber sido afectada, desde hace más de 500 años, por catástrofes periódicas, inundaciones pluviales, hídricas, las cuales se agudizaron en los últimos 30 años debido a los efectos del cambio climático, insuficientes obras de saneamiento y protecciones urbanas, dificultades en el ordenamiento territorial, la definición de áreas de riesgo hídrico, ocupación del territorio en valles de inundación tanto del río Salado como del río Paraná, entre otras cuestiones. En este marco, los ciudadanos se han apropiado del espacio urbano y construyeron su hábitat en zonas donde están expuestos a la vulnerabilidad física, ambiental, social, con escaso conocimiento de esta situación. Por lo dicho, se priorizó desde varias instituciones académicas generar proyectos de extensión que atendieran la misma.

Desde nuestra experiencia educativa, podemos afirmar que los estudiantes de Abogacía, Trabajo Social y Ciencias Hídricas se posicionan como co-constructores de aprendizajes significativos en un proceso permanente de aprender a aprender, a hacer situado en tiempo y espacio (aquí y ahora en Santa Fe), en un proceso histórico-social que hay que transformar; es decir, a partir de situaciones problemáticas que se constituyen en el marco del cambio climático, generan riesgo físico, sociocultural, ambiental en un territorio vulnerable y vulnerado, complejo donde los actores sociales tienen que empoderarse a partir de acceder a la información, conocimiento, estrategias, dispositivos, programas y políticas para una convivencia con el riesgo. Lograr una visión comprensiva de la problemática de la gestión del riesgo en sus diversos niveles, problematizándola desde la interdisciplinariedad como concepto teórico, como acción, es un hacer que permite reconceptualizar el conocimiento pedagógico didáctico (proceso de enseñanza en la educación universitaria, estrategias, ${ }^{2}$ dispositivos, ${ }^{3}$ instrumentos, etcétera). Es dable plantear que asumimos un compromiso con la pedagogía crítica fundamentada desde la teoría crítica ${ }^{4}$ y por ello venimos desarrollando un proceso reflexivo que comprende todas sus fases: preactiva, interactiva y posactiva en esta práctica innovadora tanto para los docentes como para los estudiantes.

El Proyecto de Extensión de Interés Social "El recurso hídrico en hábitat vulnerable de la ciudad de Santa Fe" es una propuesta de extensión que tiende a la creación, a la construcción de significados, poniendo eje en la emancipación de los sujetos como ciudadanos para que asuman la conducción de procesos, proyectos individuales y colectivos. La intersectorialidad e interinstitucionalidad se manifiestan a través de la participación de distintos organismos estatales de distinta jurisdicción: a) nacional a través de las distintas unidades académicas de la UNL; b) con el Ministerio de Educación de la Provincia de Santa Fe con las escuelas secundarias de gestión pública, con el Observatorio Regional del Agua del Ministerio de Infraestructura y Transporte, con la Secretaría de Protección Civil del Ministerio de Gobierno, con el Ente Aguas Santafesinas SA; y c) municipal con la Dirección de Gestión de Riesgos. Además de la participación de representantes de asociaciones civiles no gubernamentales. De allí que se comprende la enseñanza como praxis donde se propicia la reflexión y acción transformadora multiactoral, intersectorial, interdisciplinaria. Al estar dirigida por un interés emancipador, tiende a la lucha por las injusticias y asimetrías en las relaciones intersubjetivas, convirtiendo relaciones autoritarias de poder en relaciones de autoridad compartida, al decir de Souza Santos (2005).

Se intenciona el trabajo docente hacia la aproximación crítica al concepto de sujeto comprendido como un sujeto sociohistórico, titular de derechos, dinámico, potencialmente capaz de desarrollar diferentes dimensiones de su humanidad. Es un todo complejo e integral, en el que es necesario distinguir pero no fragmentar al sujeto histórico, social, político, económico, cultural (Córdoba, Gallo, Gianotti, González y Pardo, 2006). Ese sujeto estudiante es protagonista en la construcción, ampliación, resignificación,
2) Una estrategia "elabora uno o varios escenarios posibles. Desde el comienzo se prepara, si sucede algo nuevo o inesperado, a integrarlo para modificar o enriquecer la acción" (Morin,1996:127).

3) El dispositivo "constituye una forma de pensar los modos de acción, es una respuesta a los problemas de la acción", es producto "de quién o quiénes realizan un análisis de situación, contemplan la complejidad del campo de acción y ejercitan un pensamiento estratégico (Souto, 1999:93). 4) Formación de la autoconciencia para lograr crear un proceso de construcción de significados sustentado en las experiencias personales; la finalidad es el logro de la transformación social de las desigualdades, asumiendo un compromiso con la justicia, la equidad, etc. Según Giroux (2009:1), los elementos fundamentales de la pedagogía crítica son la participación, comunicación, humanización, transformación y contextualización. 5) La acción crítica y reflexiva se inscribe en la perspectiva de contribuir a formar un profesional que sea capaz de reconstruir su experiencia, reconocer y valorar el conocimiento práctico, cotidiano en su contexto sociocultural articulado al conocimiento teórico y el desarrollo de un aprendizaje más ligado a la experiencia por la vía de la investigación. 
profundización de su propio proceso de conocimiento, en tanto ser social situado, histórico, reflexivo, creativo, colaborativo, en tanto construcción participativa del conocimiento desde una relación simétrica entre docentes y estudiantes sustantivamente crítica. ${ }^{5}$ Es una propuesta de generación de los llamados conflictos cognitivos $^{6}$ para la posterior producción de síntesis en los saberes como producto de los conocimientos y experiencias.

La acción comunicativa desarrollada por los estudiantes en su trabajo con los actores institucionales en el ámbito educativo medio imprime a sus discursos el poder instituyente, al decir de Pruzzo (1998), en la medida en que sus aportes permiten ponderar desde el aprendizaje sus construcciones y mapas mentales, las diversas maneras de articular saberes, conocimientos y experiencias a las nuevas instancias de aprendizaje situado en el aquí y ahora, en la ciudad de Santa Fe, su terruño, su territorio. ${ }^{7}$ En este caso en particular, el abordaje fue sobre riesgo hídrico y en este proceso de construcción colectiva se incorpora y analiza la percepción social del riesgo como insumo para ese desarrollo.

¿Qué se pretende lograr en el proceso? A través de las pautas de trabajo interdisciplinario se accede a una práctica novedosa e innovadora para conocer una problemática de gestión del riesgo, a la comprensión; el enriquecimiento de sus estructuras cognitivas mediante la construcción de marcos conceptuales integradores de nuevos conocimientos; incrementar sus habilidades para analizar, reelaborar y resolver problemáticas conjuntas; la comprensión de los marcos teóricos y la destreza para utilizar instrumentos y técnicas de indagación, desarrollo de estrategias en el territorio, reconocimiento de la importancia del trabajo en grupo de formación donde se aporta a la integración interdisciplinar y en el mismo se enriquece la estructura sintáctica y semántica de cada disciplina, entre otras cuestiones.

El reconocimiento y la legitimación de las prácticas desarrolladas en el proyecto contribuyen a sostener la apuesta de construir comunidades de atención mutua en las que todos los participantes se ven a sí mismos como miembros de un nosotros con propósitos e intenciones compartidos.

Implica la mejora en las disposiciones y capacidades sobre la base de compromisos recíprocos que se construyen en los mismos procesos de trabajo. Implica prestar atención a la forma de situarse en la relación con otros, ya que los mismos modelan en sus prácticas una cierta valoración y confirmación mutua. Se impone entonces la necesidad de tiempos, de espacios, de voz: de un discurso con pretensión de validez donde cada uno debate y argumenta, disiente y consiente, donde se siente escuchados activamente por otros. Discurso que sugiere, invita, construye vínculos... los de los sujetos con los sentidos y significados de la experiencia realizada.

Se reconoce en esta experiencia la aproximaciones que realizan los estudiantes a la formación de un habitus ${ }^{8}$ que supone un saber hacer que se trabaja explícitamente, se lo tematiza, se lo define, se acepta que es un tema que no le corresponde únicamente a una determinada formación disciplinar y se lo vincule a un trabajo transformativo concreto en el territorio como plantea Svarzman, citado por Travi:

"el acercamiento a la subjetividad de las personas que viven día a día con el problema fue uno de los momentos más importantes. [Para enseñar hacer algo] no basta con decir cómo se hace, es imprescindible transitar por la intransferible experiencia de hacerlo con nuestras propias manos, nuestros propios saberes, nuestras experiencias anteriores, nuestras ideas acerca de la mejor manera de proceder, de actuar para operar". (2007:3)

Es en este interjuego donde se entrelazan dinámicamente lo individual lo grupal, la relación teoría-práctica, la metodología acciónreflexión-acción, las representaciones del poder y saber disciplinar, la tensión subordinación-complementariedad de los saberes.

Tal como expresan los estudiantes que participaron en los proyectos: "Comprender de forma más abarcadora el problema o el objeto de conocimiento, conlleva un diálogo abierto con cada disciplina, así como problematizar y valorar los diferentes puntos de vista", "desde mi carrera de ingeniería, no tenemos en cuenta lo social que hace al entorno de una obra, y fue bueno incorporar esa mirada". La integración disciplinar ha contribuido al afianzamiento de ciertos valores, flexibilidad, confianza, paciencia, intuición, pensamiento divergente, sensibilidad hacia las demás personas, aceptación de los riesgos, aprender a moverse en la diversidad, aceptar nuevos roles, entre otros. El saber ser, estar y valorar implica el despliegue de valores, actitudes y normas que rigen dicho ejercicio.
6) Cuando hablamos de conflicto cognitivo nos referimos en primer lugar a la confrontación mental que vive el estudiante entre su formación basada en un planteamiento teórico-metodológico centrado en la enseñanza y la experiencia centrada en el aprendizaje. Se genera cuando el estudiante adquiere conciencia de que la intervención del docente en el aula sigue una línea facilitadora, no directiva, y deben asumir mayor responsabilidad, autonomía, iniciativa y toma de decisiones (Ontoria Peña, Rubio y Sánchez, 1996:1). 7) El conocimiento situado es la visión en la que la posición desde la cual se mira define las posibilidades de lectura y acción, permite establecer conexio- nes parciales con otros agentes para construir conocimiento poniendo en tensión los conceptos de semejanza y diferencia, saber y poder, rescatando en todo momento los saberes del agente social que produce las prácticas, su proceso de producción y reproducción.
8) La construcción de un modus operandi o habitus científico es iluminador, en la medida que plantea un "proceder mediante indicaciones prácticas o correcciones aplicadas a la práctica en curso y concebidas conforme al espíritu mismo de la práctica" (Bourdieu y Wacquant, 1995:18). 
En este despliegue de valores afinados, discutidos, afianzados, en el proyecto se propicia la creación, la elaboración de un producto escrito que refleje el trabajo interdisciplinario, interinstitucional e intersectorial sobre una problemática propia y común a muchos, como ejercicio de la ciudadanía de los estudiantes de las distintas disciplinas y niveles educativos.

\section{Conclusiones}

Las novedosas relaciones construidas por los sujetos del proceso de enseñanza-aprendizajes, las relaciones docentes-estudiante desde un posicionamiento teórico epistemológico y pedagógico crítico, de diálogo en la construcción conjunta del conocimiento y la participación democrática en el desarrollo de un enfoque interdisciplinar, son fundantes para el conflicto, el cambio conceptual que permiten la construcción de significados y conceptos de los sujetos en el nivel superior de la enseñanza. Es por ello que desde el proyecto "El recurso hídrico en hábitat vulnerable de la ciudad de Santa Fe" se comprende el proceso en constante resignificación dinámica de todos los componentes: objetivos, contenidos, metodologías, estrategias, dispositivos que van de la planificación a la evaluación integral, identificación y recreación de formas de desarrollar la enseñanza interdisciplinaria. Las innovaciones incorporadas con aportes metodológicos de la pedagogía crítica y el constructivismo posibilitaron que los estudiantes se apropiaran de modos de aprender que revalorizan el sentido común de los actores sociales en su vida cotidiana como conocimiento práctico incorporándolo como válido. En este proceso de identificación de diversos problemas complejos que atraviesan la problemática hídrica, programar, ejecutar estrategias de intervención (talleres participativos, utilización de mapeos dinámicos e interactivos para reconocimiento del territorio, ubicación de actores sociales, inventario de recursos y servicios, videos, entre otros), hizo factible incorporar nuevas tecnologías, recrearlas y complementarlas integralmente y fortalecer el proceso de formación profesional.

Consideramos que los proyectos ejecutados han impactado sustantivamente al interior de las ciencias: mostraron que es posible la articulación concreta, proactiva y creativa de las mismas para modificar los modos de entender y accionar en nuestro territorio, vulnerable a las emergencias hídricas.

Estos proyectos se constituyeron en espacios de prácticas de formación profesional de la Licenciatura en Trabajo Social y Abogacía, lo cual permitió hasta el momento, una mayor articulación entre formación y extensión.

En este sentido reafirmamos el propósito y el compromiso de las universidades de afianzar los mecanismos para contribuir a formar profesionales comprometidos con la gestión de riesgo en un territorio vulnerado y vulnerable, capaces de problematizar y tensionar, miradas, herramientas, dispositivos que, favorezcan el despliegue de una actitud abierta, flexible hacia el trabajo interdisciplinario, interactoral, intersectorial, desde un posicionamiento ético-político comprometido con los desafíos de la realidad local, regional, nacional y latinoamericana.

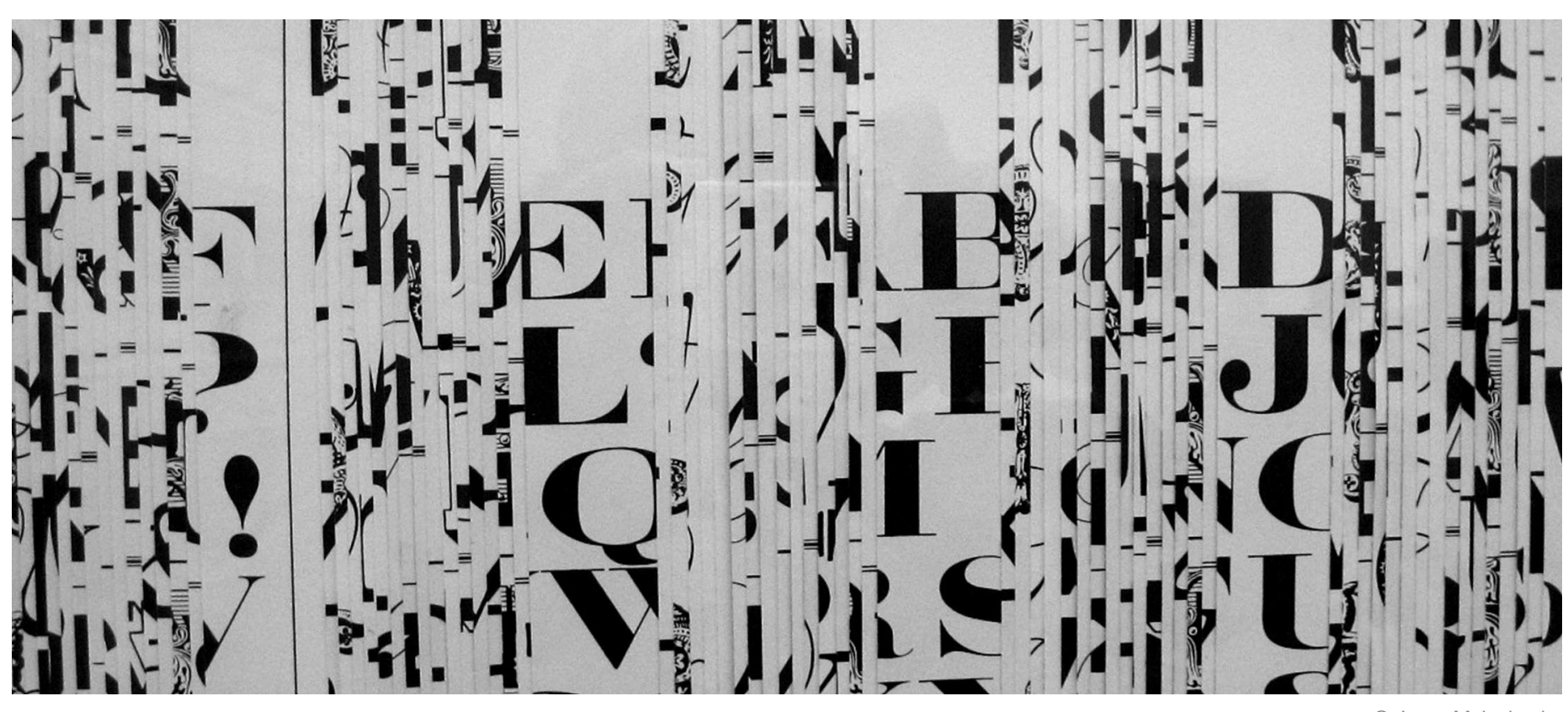

(C) Jorge Malachesky 


\title{
66
}

\author{
las innovaciones incorporadas \\ con aportes metodológicos \\ de la pedagogía crítica y el \\ constructivismo posibilitaron \\ que los estudiantes se \\ apropiaran de modos de \\ aprender que revalorizan el \\ sentido común de los actores \\ sociales en su vida cotidiana
}

\section{Referencias bibliográficas}

Apostel, L., Briggs, A. y Michaud, G. (1979). Interdisciplinariedad. Problemas de la enseñanza y de la investigación en las universidades. México: ANUIES.

Arias Alpízar, L. (2009). Interdisciplinariedad y triangulación en Ciencias Sociales. Diálogos Revista Electrónica de Historia, 10 (1, febrero-agosto), 120-136. Universidad de Costa Rica.

Bourdieu, P. y Wacquant. L. (1995). Respuestas para una Antropología comprensiva. Introducción de Loïc Wacquant. México: Grijalbo. $1^{\circ}$ edición.

Carballeda, A. (2007). La interdisciplina como Diálogo. Una visión desde el campo de la Salud, 1-5. Portal Margen. Recuperado de http://www.buenosaires.gob.ar/ areas/salud/dircap/mat/matbiblio/carballeda.pdf (17/06/2016).

Córdoba, A. (2007a). Los procesos reflexivos en la enseñanza del trabajo social. Aportes para la construcción del pensamiento autónomo. Ponencia presentada al II Encuentro Argentino y Latinoamericano "Prácticas sociales y Pensamiento Crítico. Escuela de Trabajo Social. UNC. Córdoba, 4 y 5 de julio.

Córdoba, A. (2007b). La construcción del conocimiento situado en los procesos de desarrollo profesional en el campo social. Aportes y reflexiones desde la práctica de la enseñanza en el postítulo: especialización en abordaje integral de problemáticas sociales en el ámbito comunitario de la Universidad de Lanús - Ministerio de Desarrollo Social de la Nación. XXIV Congreso Nacional de Trabajo Social. Mendoza. 4, 5 y 6 de octubre.

Córdoba, A.; Gallo, S.; Gianotti, M.; González, A. y Pardo, M. (2006). Universidad y desarrollo del hábitat social. Articulación con los actores de la gestión habitacional. Desafíos para la formación desde el concepto de producción social del hábitat. XII Encuentro de la Red ULACAV. Red Universitaria Latinoamericana de Cátedras de Vivienda. Mendoza-Argentina, 5 al 7 de octubre.

Del Percio, E. (2010). Complejidad e indisciplina. Cuando la epistemología se cruza con la política. Conferencia Especial pronunciada en el marco del VI Congreso Mundial de la Complejidad. La Habana, 8 de enero.

Ferry, G. (1997). Pedagogía de la formación. Novedades Educativas. Colección Formación de Formadores. Buenos Aires: Facultad de Filosofía y Letras, UBA. $1^{\circ}$ edición.

Follari, R. (2008). Relevo en las ciencias sociales latinoamericanas. Estudios culturales transdisciplinariedad y multidisciplinariedad. Diálogos de la comunicación. Federación Latinoamericana de Facultades de Comunicación Social. http://dialogosfelafacs.net/wp-content/uploads/2015/63/RELEVO-EN-LAS-CIENCIAS-SOCIALES-LATINOAMERICANAS.pdf (14/6/2016).

Giroux, H. (2009). Los profesores como intelectuales. Madrid: Paidós.

Morin, E. (1996). Introducción al pensamiento complejo. Barcelona: Gedisa. $2^{\circ}$ edición.

Morin, E. (2004). Los sietes saberes necesarios para la educación del futuro (Introducción). Buenos Aires: Nueva Visión. $1^{\circ}$ edición.

Ontoria Peña, A.; Molina Rubio, A.; Luque Sánchez, Á. (1997). Autoconciencia del conflicto cognitivo ante la innovación metodológica en la formación inicial de maestros. Revista Electrónica Interuniversitaria de Formación del profesorado, 1(0). Asociación Universitaria de Formación del Profesorado (AUFOP). VIII Congreso de Formación del Profesorado. Ávila.

Piaget, J. (1970). Psicología, lógica y comunicación. Buenos Aires: Nueva Visión. Pruzzo de Di Pego, V. (1998). Evaluación curricular: evolución para el aprendizaje (Introducción). Buenos Aires: Espacio Editorial. Colección Didáctica. $1^{\circ}$ edición. Souto, M.; Barbier, J.; Cattaneo, M.; Coronel, M.; Gaidulewicz, L.; Goggi, N.;

Mazza, D. (1999). Grupos y dispositivos de formación (Introducción). Buenos Aires. Novedades Educativas - Facultad de Filosofía y Letras, UBA. Serie Formación de formadores. $1^{\circ}$ edición.

Sousa Santos, B. de (2005). La universidad del siglo XXI. Para una reforma democrática y emancipatoria de la Universidad. Palabras preliminares a esta edición: Daniel Cazés Menache. Centro de Investigaciones Interdisciplinarias en Ciencias y Humanidades. Coordinación de Humanidades. México. Universidad Autónoma de México. Colección Educación Superior.

Travi, B. (2007). El proceso de enseñanza-aprendizaje de los contenidos procedimentales en la asignatura Trabajo Social II de la Carrera de Licenciatura en Trabajo Social de la Universidad Nacional de Luján (Unlu). Boletín Electrónico Sura. Universidad Costa Rica. Mimeo. 